\title{
libMCXray: A Monte Carlo Simulator for Signal Analysis inside Dragonfly Software
}

\author{
S. Rudinsky, ${ }^{1}$ M. Gendron, ${ }^{2}$ N. Piché,${ }^{2}$ M. Marsh, ${ }^{2}$ and R. Gauvin ${ }^{3}$ \\ ${ }^{1}$ Steam instruments, 931 E. Main St., Ste. 3, Madison, WI, 53703-2955 \\ ${ }^{2}$ Object Research Systems, 101-760 Rue Saint-Paul Ouest, Montreal, QC, H3C 1M4 \\ ${ }^{3}$ McGill University, 3610 University, Montreal, QC, H3A 0C5 \\ sam.rudinsky@steaminstruments.com
}

\begin{abstract}
Monte Carlo simulations are commonly used in elemental quantification using energy-dispersive spectroscopy (EDS). Here, the Monte Carlo program MC X-ray was incorporated into the image processing software Dragonfly by Object Research Systems (ORS) as a simulation library. The simulation program has been transformed into a complete microscope simulator where the tools of Dragonfly allow complex voxel-based geometries to be constructed, and the electron beam and detectors can be freely placed inside the 3D space. Computation times of simulations have been improved drastically through new data structures and parallelization. Simulations of backscattered electron imaging and EDS mapping are presented here to demonstrate the capabilities of this new library.
\end{abstract}

Keywords: scanning electron microscopy, energy-dispersive spectroscopy, segmentation, Monte Carlo simulations, Dragonfly software

\section{Introduction}

Monte Carlo simulations are widely used in applications related to both electron beam imaging and microanalysis [1]. They provide information such as the interaction volume of incident electrons and X-ray distributions [2], and they are useful in cathodoluminescence emission measurements [3] and mass thickness estimations $[4,5]$. They also compute backscattered and secondary electron yields, which are important for image simulations used to estimate signal variations [6,7] and secondary election emissions of electrode materials [8]. Finally, a crucial use of Monte Carlo simulations is to perform accurate elemental quantification from energy-dispersive spectroscopy (EDS) data. Matrix correction factors can be computed using Monte Carlo simulations and then used to calculate accurate compositional information from X-ray intensities [9-11]. This allows quantification to be done without the need for standards or pre-measured experimental databases [10].

The most widely used programs available are PENEPMA, CASINO, and DTSA-II [12-14]. While these programs have been well adapted for common uses in electron microscopy and microanalysis, there exist some limitations to their usability. The most notable of these are the computation time and restrictions on sample and microscope geometries. For example, simulations of electron numbers on the order of $10^{5}$ at a single point may take approximately a minute on a desktop computer [15]. These long computing times can make simulations of backscattered electron (BSE) images or EDS maps unfeasible in a reasonable amount of time. The limitations in terms of geometry impact the usability of these programs on complex materials. Important obstacles arising from non-flat sample geometries can strongly impact accurate elemental quantification [16,17]. To simulate such materials, freedom in terms of sample and microscope geometry and placement must be possible.

Here, we present the incorporation of the existing Monte Carlo software MCX-ray [18] into the image processing software
Dragonfly developed by Object Research Systems (ORS) Inc. [19]. The class system of Dragonfly allows substantial freedom in terms of sample geometry and placement. Furthermore, additions have been made to turn MC X-ray into a full microscope simulation tool capable of reproducing some elements of an electron microscope. Multiprocessing has also been implemented to decrease computing times substantially, allowing more complex problems to be easily evaluated. The manuscript is structured as follows. First, the details and usability of the program are described with reference to previous Monte Carlo software. Then applications of the library to BSE image simulations and elemental quantification are presented. Finally, some concluding remarks are made about the method.

\section{Materials and Methods}

MC X-ray is a Monte Carlo-based simulation package [18] that was devised as an extension of CASINO $[13,20]$ and Win $\mathrm{X}$-ray [21], specific for X-ray microanalysis. Scattering events are modeled based on a stochastic process where electrons are simulated using a forward scattering random walk. An electron is initiated, and uniform random numbers are generated and used in cross-sectional models to determine the particle's path through the sample material. The physical models, methodology, and calculations used are described in other work [21]. The previously stand-alone MC X-ray program was incorporated into the image processing software Dragonfly as a feature plug-in. The base code was written in $\mathrm{C}+\mathrm{H}$, and the interface used to gather the input and call the simulation functions was written in Python 3.

Sample generation is done using the Multi-region of interest (ROI) class inside Dragonfly. A phantom material is created as a MultiROI where each subgeometry is its own ROI. The structures are voxel-based where the dimensions of the voxels are chosen by the user. Once a phantom has been defined, labels may be assigned to each ROI. Each label contains a property where, for the purpose of libMCXray, these properties represent the constituent elements of each region. The labels are vectors whose lengths are the number of regions and whose values are the composition of the elements in the indexed region. Thus, a material may be generated with a multitude of elements in a variety of regions with varying compositions. MultiROIs can be created by the user or generated from other image data such as stacks of images, which can be compiled into a 3D structure.

The simulation environment comprises an electron beam, a sample material, and a series of detectors. The electron beam is above the sample material normal to its surface. The user input parameters of the beam are the incident electron energy, incident current, and working distance. The rasterizing across the sample surface is done by displacing the beam across the 
Table 1: EDS detector parameters modifiable by user in libMCXray.

\begin{tabular}{|l|}
\hline Input properties for simulated EDS detector \\
\hline Crystal type (Al or Si) \\
\hline Crystal elemental density \\
\hline Crystal dimensions (radius and thickness) \\
\hline Dead layer thickness \\
\hline Diffusion length \\
\hline Detector efficiency \\
\hline Take-off angle of detector \\
\hline Position of detector in space \\
\hline Number of energy channels \\
\hline
\end{tabular}

sample surface at steps specified by the user input resolution. Once the sample material is constructed, it is placed so that the beam and the sample are along the same axis. Their vertical distance is specified by the desired working distance.

Detectors may then be added to the simulator. The BSE detector may be defined as a disc or circular annulus below the electron gun. The input in this case is the inner and outer radius of the annulus or the radius of the disc. BSEs are defined as those which have exited the sample and intersected the detector. The EDS detector is defined by the properties specified in Table 1 and are similar to the stand-alone version of MC X-ray. Multiple detectors can be added to the simulator, each with their own properties. Last, bright-field and darkfield detectors can be added below the sample. Each is defined by their distance from the exit surface of the sample and their inner and outer solid angles. Again, intensities of either signal are constructed from the number of electrons intersecting the respective detectors upon exit from the sample.

The program output is separated into two parts: electron and X-ray data. From the rasterizing, the BSE image and any

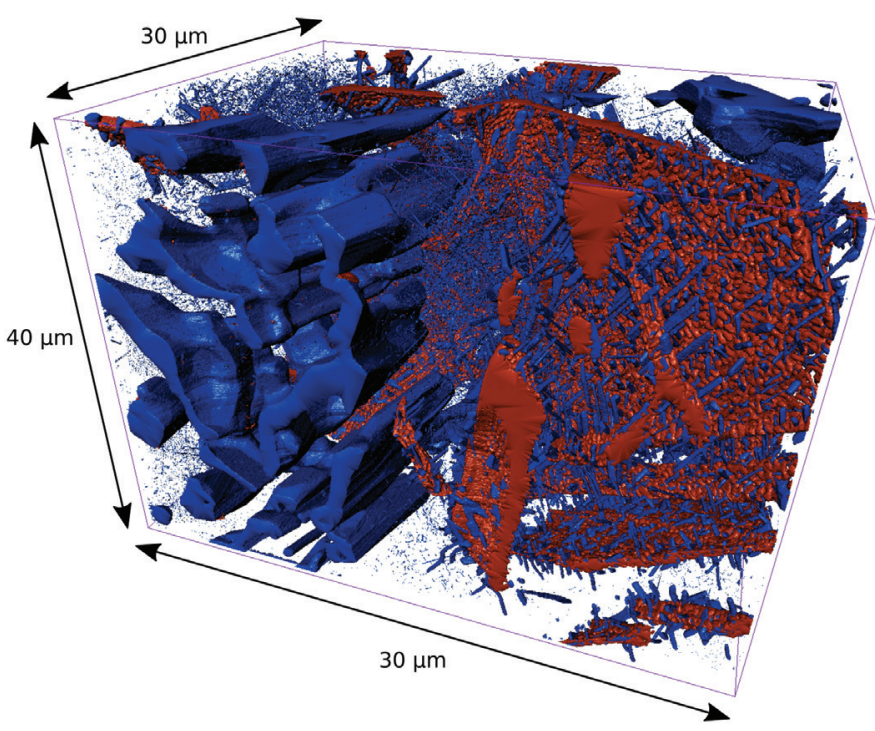

Figure 1: 3D representation of sample geometry used in simulation experiment. Phase 1 is the matrix, phase 2 is in red, and phase 3 is in blue. The matrix phase is transparent.
Table 2: Compositions of the simulated system assigned to the MultiROI of Figure 1.

\begin{tabular}{|l|c|c|}
\hline Phase number & Element & Color in Figure 1 \\
\hline 1 & $\mathrm{Ni}$ & Transparent \\
\hline 2 & $\mathrm{Fe}$ & Red \\
\hline 3 & $\mathrm{Cr}$ & Blue \\
\hline
\end{tabular}

possible transmitted signal images are output as 2D arrays of floating-point real numbers. The EDS maps are output as 3D arrays where the first two dimensions are the point coordinates and the third dimension is the energy spectrum. Finally, if point analyses are chosen, the X-ray depth distribution curves, $\phi(\rho z)$, of specified transitions may be output.

\section{Results and Discussion}

BSE image simulation. A MultiROI of a three-phase alloy compiled from a focused ion beam image dataset was used to simulate backscattered images. A 3D perspective of the MultiROI is depicted in Figure 1. Elemental compositions were assigned to the phases prior to performing the Monte Carlo simulation. A Ni-Fe-Cr system was investigated where each element was associated to a pure single phase. The phases to which each element is associated are presented in Table 2 . The incident probe position was rasterized across the top surface of the sample shown in Figure 1. The sample plane at the incident direction is displayed in Figure 2.

Simulations were performed at $15 \mathrm{keV}$ using 5,000 electrons. Because the simulations of each probe position are independent, the method is easily parallelized, allowing for an acceleration of 80 to 100 times the computing time of the original program. The simulated BSE image corresponding to a subsection of the incident plane of Figure 2 is presented in Figure 3.

The image consists of $512 \times 512$ pixels situated about the center of the plane. This area is indicated in Figure 2 by a dashed rectangle. Here, the three pure element phases are clearly distinguished with the fine structures from the underlying geometry clearly visible. The differences in signal level clearly reflect

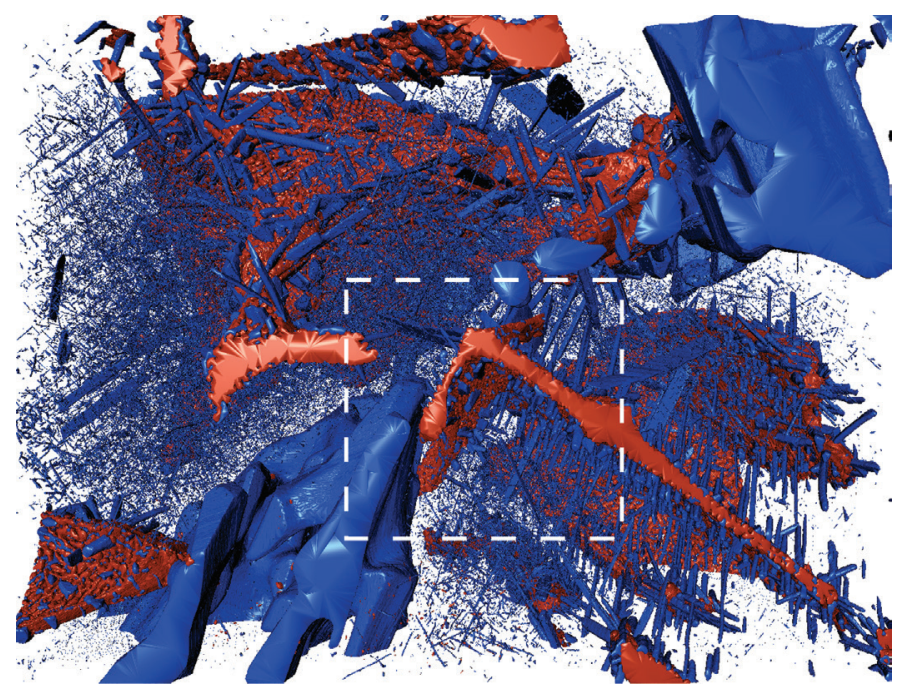

Figure 2: View of simulated material from beam incident direction. The area across which the beam was rasterized is indicated by a dashed rectangle. 


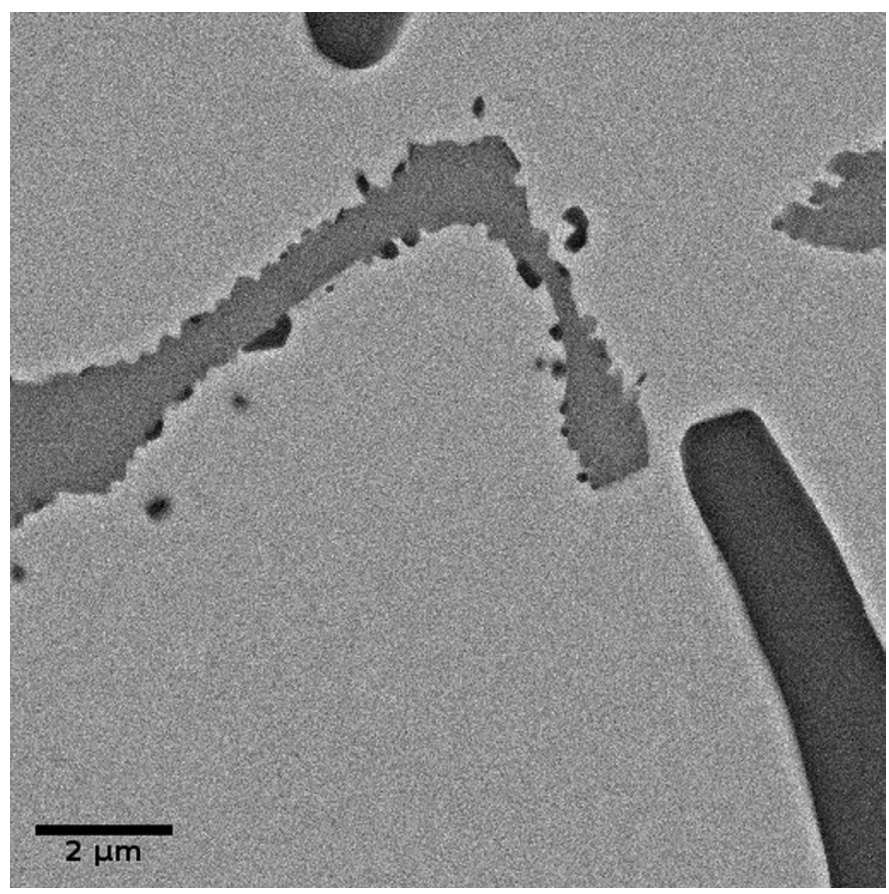

Figure 3: Simulated BSE image of three-component $\mathrm{Ni}-\mathrm{Fe}-\mathrm{Cr}$ system at 15 keV using libMCXray.

the differences in atomic number between each phase. The matrix is composed of pure $\mathrm{Ni}$, the heaviest of the three elements, whose signal level is the highest. In comparison, the $\mathrm{Cr}$ phase, contained in the majority of the needle-like structures, is seen as the phase containing the lowest level of signal, consisting of points attached to the Fe phase and the large rectangular structure in the bottom right corner of the image.

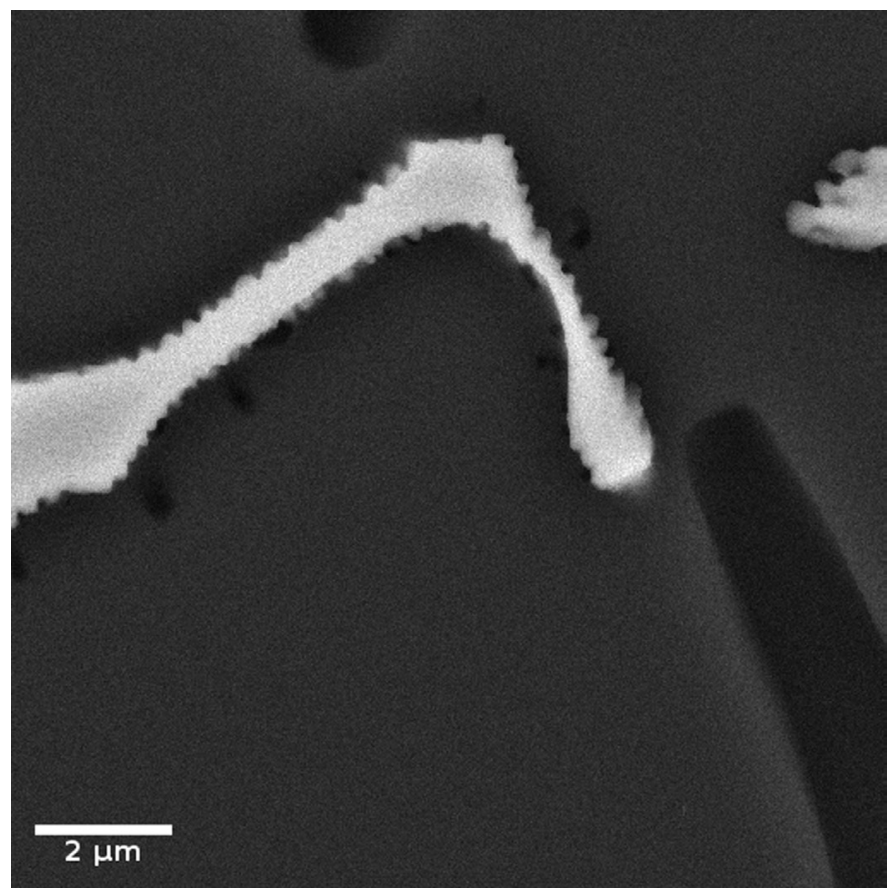

Figure 4: Simulated BSE image of Al-Si alloy containing three phases: pure $\mathrm{Al}, \mathrm{Al}_{2} \mathrm{Cu}$, and $\mathrm{Mg}_{2} \mathrm{Si}$.

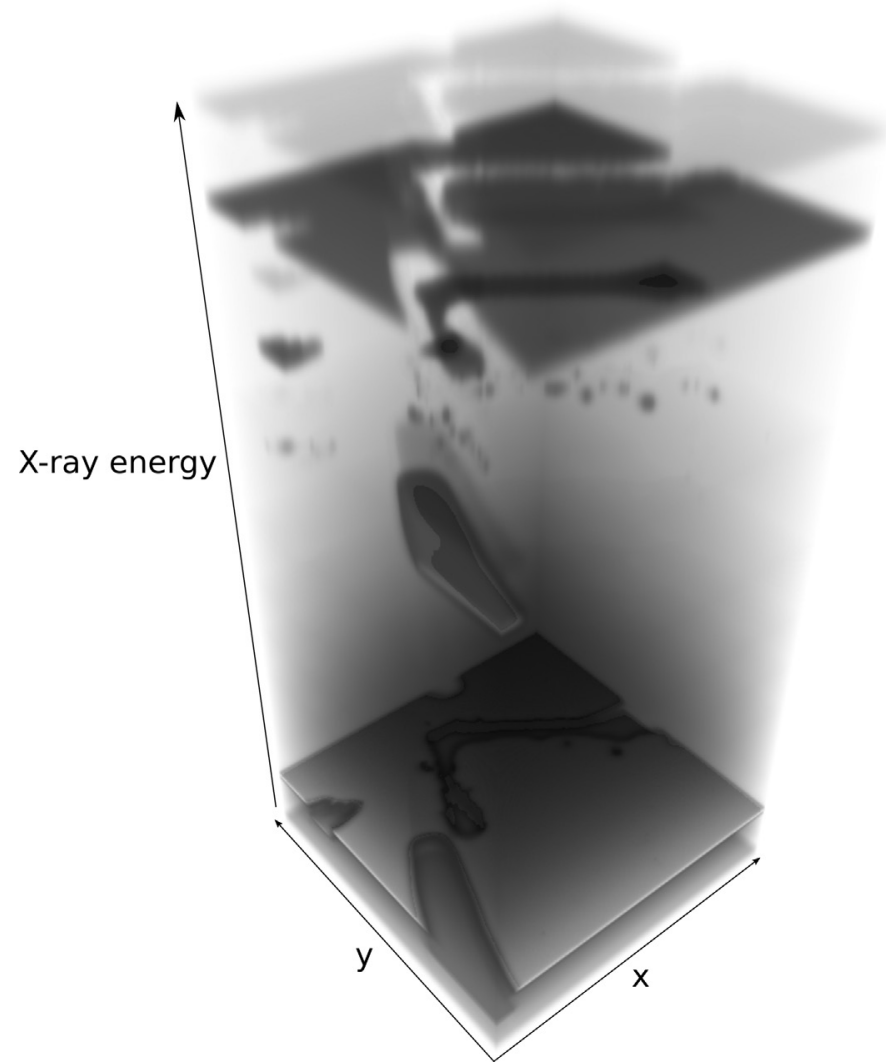

Figure 5: Three-dimensional depiction of the EDS dataset simulated using libMCXray. The image represents spatial dimensions of $512 \times 512$ pixels and 2048 energy bins.

Another system was simulated consisting of the expected phases of an Al-Si alloy to show the versatility of the method in simulating alloys closer to reality. Figure 4 shows a simulated BSE image of the same geometric structure, however here the phases consisted of pure $\mathrm{Al}$ as the matrix, phase $1, \mathrm{Al}_{2} \mathrm{Cu}$ as phase 2, and $\mathrm{Mg}_{2} \mathrm{Si}$ as phase 3. Simulations were performed under the same conditions as the $\mathrm{Ni}-\mathrm{Fe}-\mathrm{Cr}$ system.

Because $\mathrm{Cu}$ has a much higher atomic number than the other constituent elements, and hence a higher probability of scattering, the signal level of the $\mathrm{Al}_{2} \mathrm{Cu}$ phase is the highest in the image. In comparison, the $\mathrm{Mg}_{2} \mathrm{Si}$ phase contains the lowest signal level due to its lower probability of scattering. This demonstrates that even multi-component phases may properly and easily be simulated using libMCXray, and the attributes of the Dragonfly software aid in proper image analysis.

EDS spectrum analysis and mapping. EDS maps were simulated for the above system using the same MultiROI and positions. Here, 2048 channels were used across the energy scale. Figure 5 shows the entire 3D dataset acquired using libMCXray, two spatial dimensions and the third in energy. The EDS simulation was performed on the Amazon AWS highperformance computing (HPC) network and took 1 hour on 500 computers each containing 8 cores. On an extremely performant workstation, such a simulation would take approximately 200 hours. Computing times are rendered much more reasonable by the use of cluster computing. 

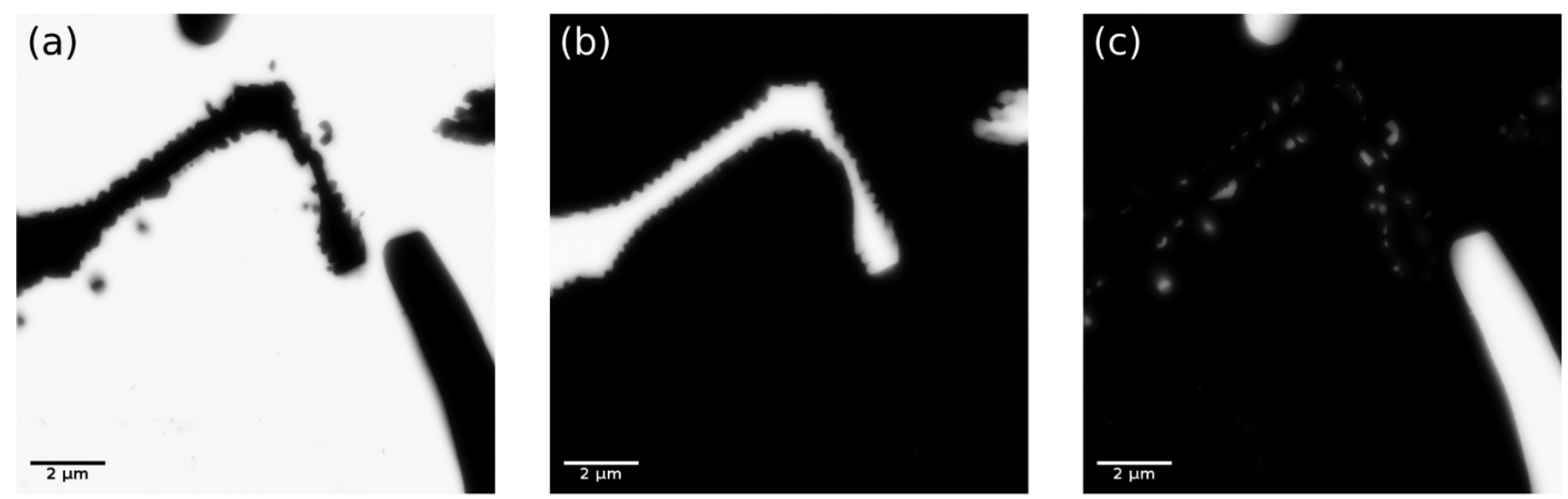

Figure 6: Simulated EDS maps for (a) Ni K $\alpha$, (b) Fe K $\alpha$, and (c) $\mathrm{Cr}$ K $\alpha$ transitions. These correspond to 7.477, 6.403, and 5.414 keV respectively.
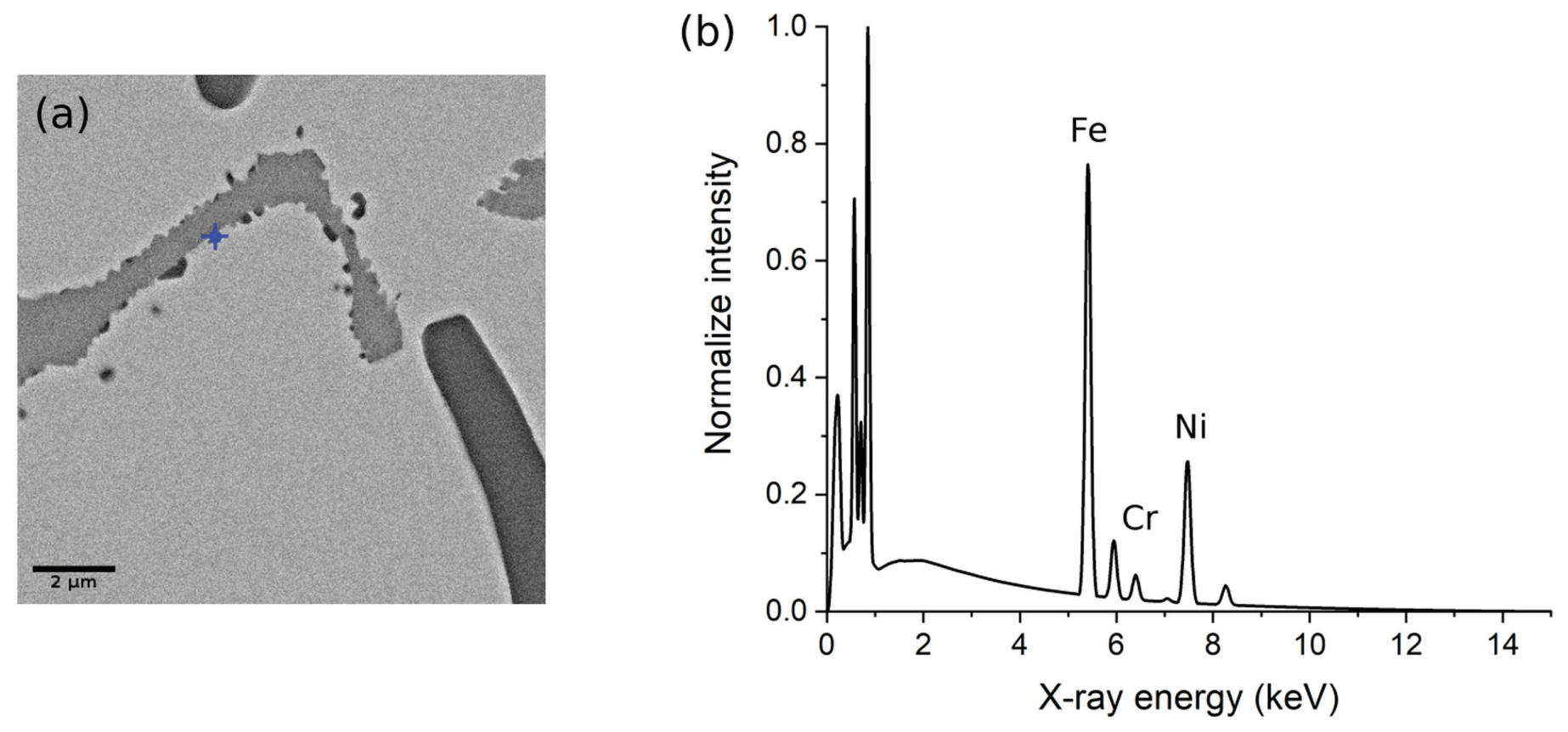

Figure 7: (a) BSE image showing the point at which an energy spectrum was taken and (b) corresponding energy spectrum showing peaks from Ni, Fe, and Cr. The labels are placed above the $\mathrm{K} \alpha$ peaks.

Slices of the dataset were taken at the corresponding $\mathrm{K} \alpha$ transition energies for each element. These slices are depicted as 2D maps in Figure 6.

The simulated EDS maps correspond exactly to the BSE image and simulated geometry. Areas of white imply areas of high intensity, while black describes areas of low to no intensity. The simulated spectrum at a point is depicted in Figure 7 along with the BSE image showing the point at which the spectrum was taken.

At this point, contributions from all three phases are present. Although these results are all simulated, the accuracy of MC X-ray simulations for K-shell ionizations has been demonstrated in previous work [22-25].

\section{Conclusions}

The Monte Carlo simulation program MC X-ray was incorporated into the Dragonfly software package by ORS to simulate BSE imaging and EDS analysis inside an allencompassing image processing software. Using parallelization and good software practice, the calculation speed was improved by $\sim 80-100$ times the original code. Furthermore, using the Dragonfly interface, complex geometries may be created or even imported from datasets such as those generated in focused ion-beam experiments. Future work will see the addition of secondary electron detection and the incorporation of higher-order ionization effects such as secondary fluorescence yields.

\section{References}

[1] CGH Walker et al., Scanning 38 (2016) 802-18.

[2] A Morozov et al., Eur Phys J D 48 (2008) 383-88.

[3] M Toth and MR Phillips, Scanning 20 (1998) 425-32.

[4] D Moro et al., Measurement 129 (2018) 211-17.

[5] A Pazzaglia et al., Mater Charact 153 (2019) 92-102. 
[6] AH Mir et al., Micron 126 (2019) 102712.

[7] MHN Yio et al., Cement Concrete Res 89 (2016) 320-31.

[8] HY Chang et al., Nucl Instrum Meth B 454 (2019) 14-22.

[9] J Donovan et al., Microsc Microanal 25(3) (2019) 735-42.

[10] P Horny et al., Microsc Microanal 16(11) (2010) 821-30.

[11] N Brodusch and R Gauvin, J Microsc 267(3) (2017) 288-98.

[12] X Llovet and F Salvat, Microsc Microanal 23(3) (2017)

[13] P Hovington et al., Scanning 19(1) (1997) 1-14.

[14] NWM Ritchie, Microscopy Today 19(1) (2011) 26-31.

[15] NWM Ritchie, Microsc Microanal 15(5) (2009) 454-68.

[16] DE Newbury and NWM Ritchie, Scanning 35(3) (2013) 141-68.

[17] DE Newbury, Scanning 26(3) (2004) 103-14.

[18] R Gauvin and P Michaud, Microsc Microanal 15(Suppl 2) (2009) 488-89.

[19] Object Research Systems (ORS) Inc., Dragonfly 3.6. Montreal, Canada, 2018. Software available at http://www. theobjects.com/dragonfly.

[20] D Drouin et al., Scanning 29(3) (2007) 92-101.

[21] R Gauvin et al., Microsc Microanal 12(1) (2006) 49-64.

[22] R Wuhrer and K Moran, IOP Conf Ser-Mat Sci 55 (2014) 012021 .

[23] MJ-F Guinel et al., J Microsc 255(3) (2014) 128-37.

[24] N Brodusch et al., Microsc Microanal 23(Suppl 1) (2017) 236-37.

[25] C Teng et al., Microsc Microanal 23(Suppl 1) (2017) 1046-47. 634-46.

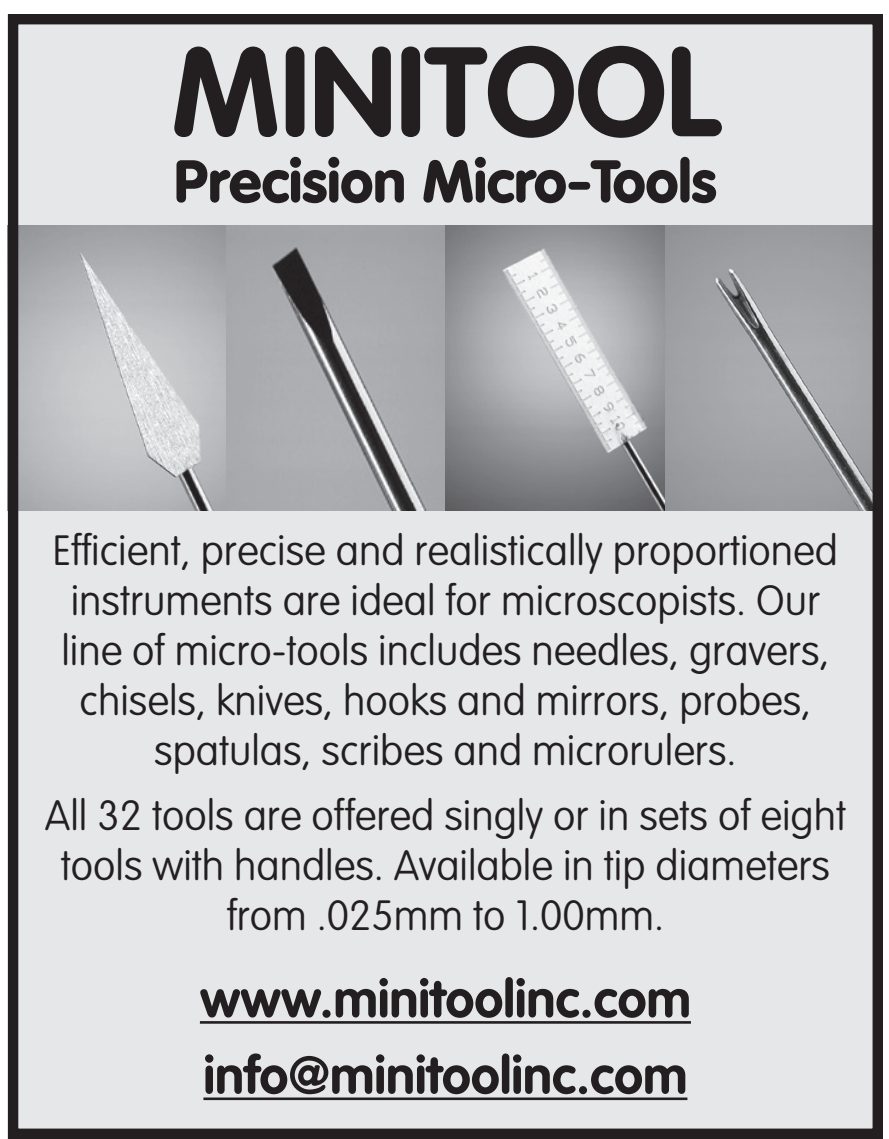

\section{MEO Engineering Company, Inc.}

High Technology on a Small Scale since 2004

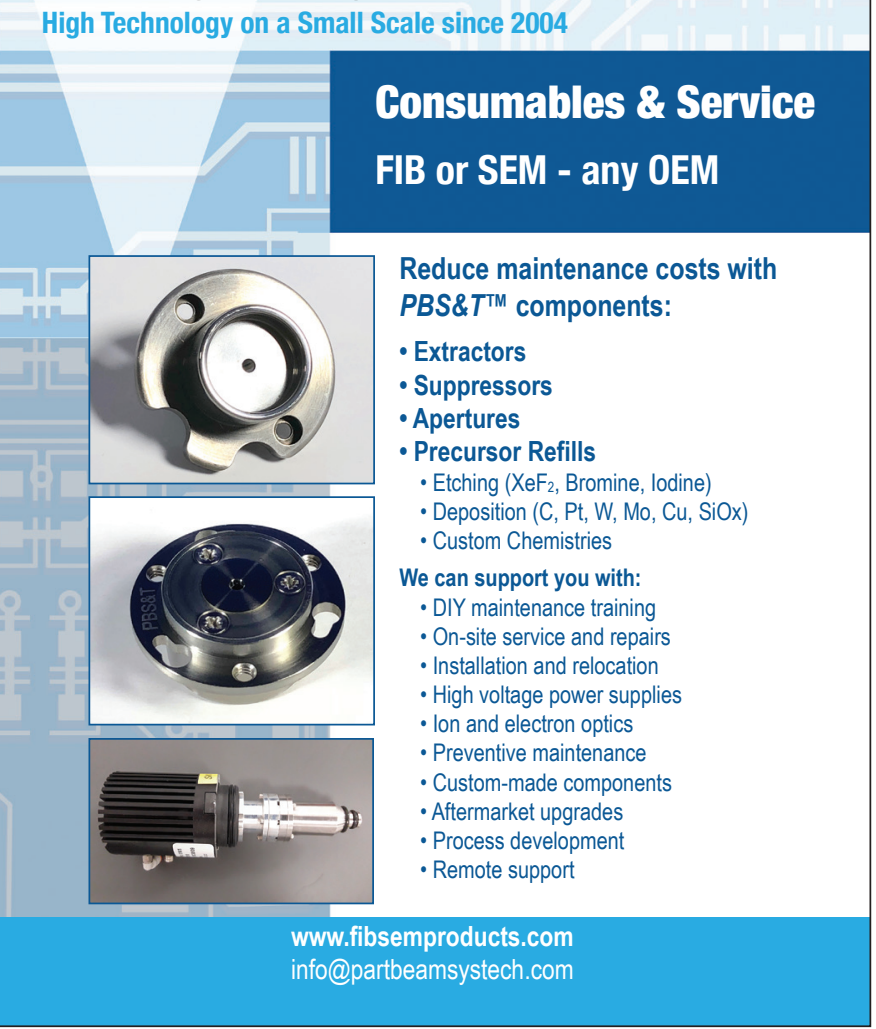

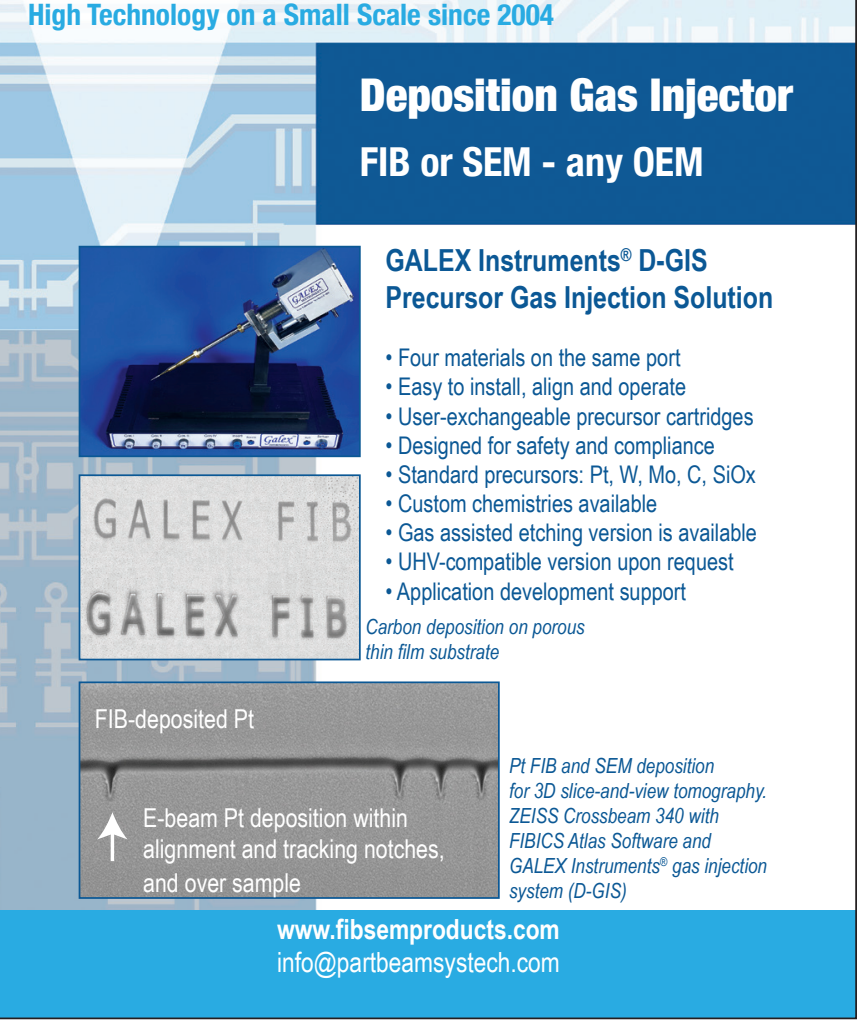




\section{PNDetector}

PNDetector's SDDs are at the forefront of radiation detector technology, as they combine both excellent energy resolution and short processing times. We are fabricating in our own cleanroom facility dedicated to the high quality fabrication of modern silicon radiation detectors and is optimized to permit a continuous and efficient production flow.

\section{High Resolution Silicon Drift Detector with superior performance}

$\checkmark$ Up to 1 Mcps for single SDDs and several Mcps with our Multi-Element SDDs

$\checkmark$ Best energy resolution down to $121 \mathrm{eV}$

$\checkmark$ Best light element detection

$\checkmark$ P/B ration up to 20.000

$\checkmark$ Ultralow leakage current $<100 \mathrm{pA} / \mathrm{cm}^{2}$

$\checkmark$ Radiation hardness $>10^{14}$ photons $/ \mathrm{cm}^{2}$

$\checkmark$ Wide selection of chip sizes and detector housings

\section{Backscattered Electron}

\section{Detector}

is designed to fulfil any customers wish from regular needs up to highly specific demands like high speed imaging or low noise signal detection at a broad range of electron energies.

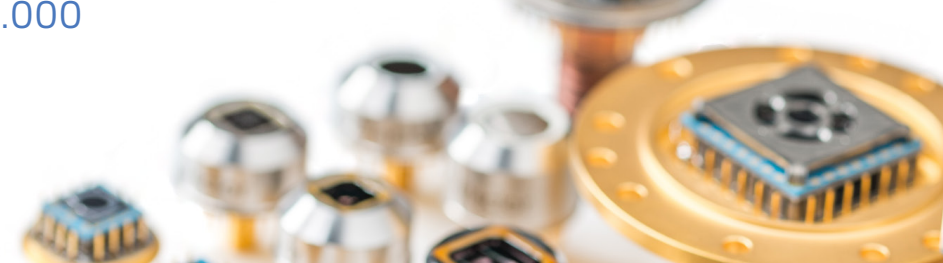

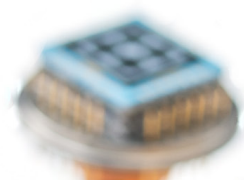

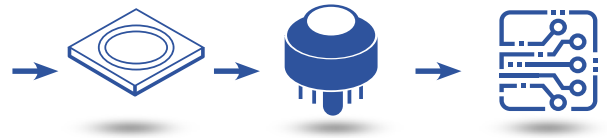

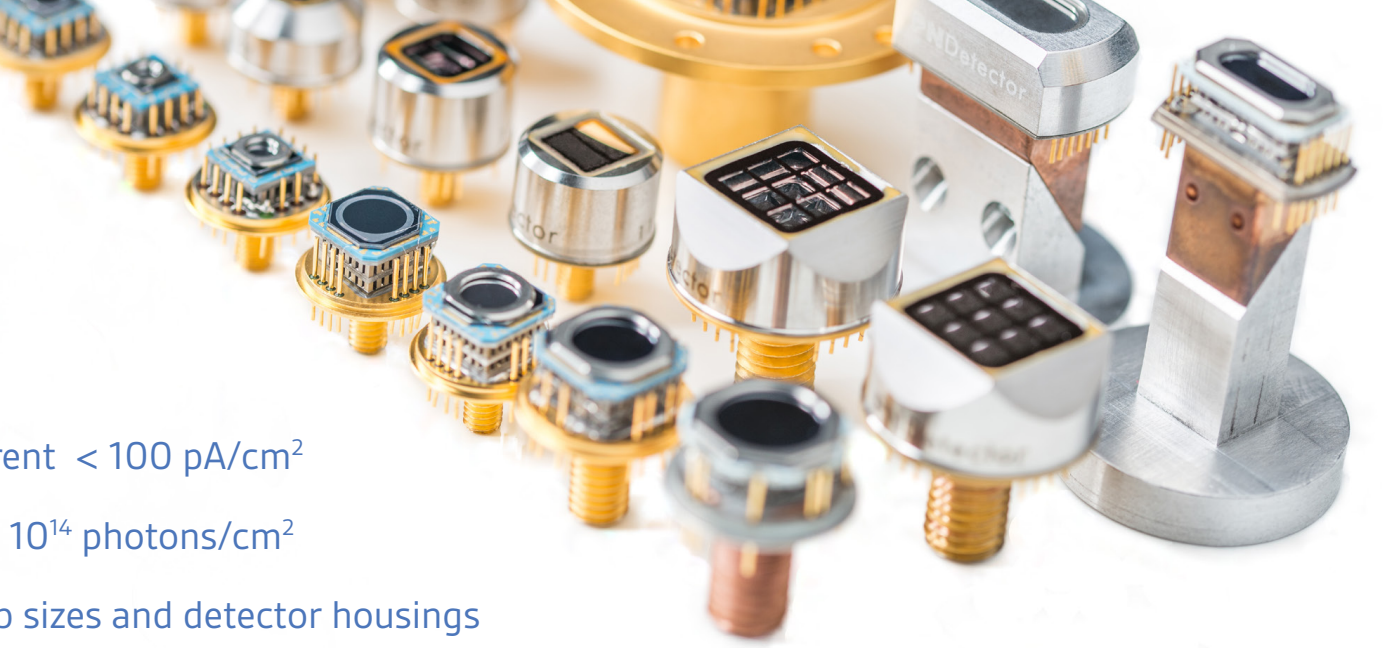

pnCCD 4D (S)TEM Camera

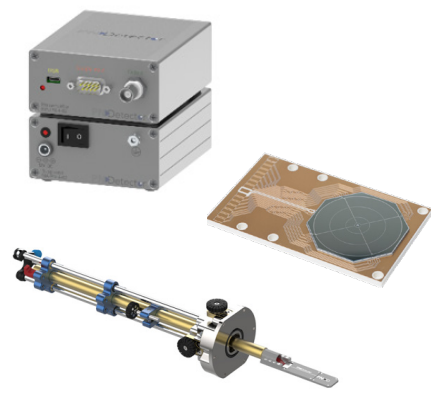

is a pixelated, ultrafast (up to $7.500 \mathrm{fps}$ ), and direct detecting electron sensor for applications in TEM and SEM. Thanks to its outstanding signal to noise ratio, single primary electron detection from

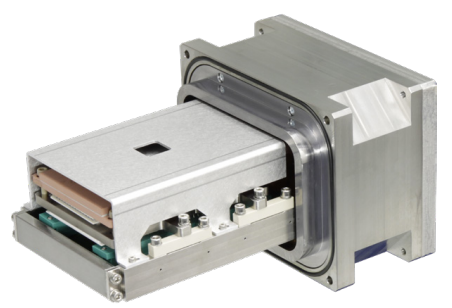
$20 \mathrm{keV}$ to $300 \mathrm{keV}$ is possible. 